\title{
Marcumar-Patient
}

\section{Unter Cotrimoxazol steigt die Blutungsgefahr}

In Harnwegsinfektionen sind ein häufiges Leiden bei älteren Menschen. Bei der Wahl des Therapeutikums sollte man gerade bei Patienten jenseits der 60 darauf achten, ob sie einen Vitamin-K-Antagonisten einnehmen. Wie eine kanadische Studie zeigte, erhöht das bei Harnwegsinfektionen mit am häufigsten verschriebene Antibiotikum Cotrimoxazol in Komedikation mit Warfarin das Blutungsrisiko.

Im Rahmen der Studie wurden 134637 Warfarinpatienten im Alter von 66 Jahren und darüber erfasst, von denen 2151 wegen einer Blutung im oberen Gastrointestinaltrakt stationär behandelt wurden. Das Risiko für diese Komplikation war unter Co- trimoxazol vierfach höher. Ein erhöhtes Risiko fand sich auch unter Ciprofloxacin (RR 1,9), aber nicht unter anderen Antibiotika. Cotrimoxazol hemmt das an der Metabolisierung von Warfarin und Phenprocoumon beteiligte Cytochrom P450 2C9.

Studienautor Hadas D. Fischer riet bei der kritischen Konstellation dazu, auf ein anderes Antibiotikum auszuweichen. Falls kein passendes Alternativpräparat infrage komme, so Fischer, sei ein straffes Monitoring der Antikoagulation sowie eine zeitweise Reduktion der Warfarindosis erforderlich.

BS II

\section{Arch Intern Med 2010;170:617-621}

\section{MELDUNGEN}

\section{HERZINSUFFIZIENZ}

\section{Ventrikelrekonstruktion ist out}

Die Herzchirurgen haben einen operativen Irrweg aufgedeckt: die Rekonstruktion des linken Ventrikels bei Patienten mit fortgeschrittener Herzinsuffizienz zusätzlich zum Bypass. Die STICH-Studie (Surgical Treatment for Ischemic Heart Failure) zieht nach vier Jahren Follow-up einen Schlussstrich: Die Ventrikelrekonstruktion bringt im Hinblick auf die Überlebenszeiten keinen prognostischen Vorteil. Die Daten der STICHStudie wurden an 595 Patienten erhoben, aus Deutschland nahm lediglich die Freiburger Gruppe um Prof. Beyersdorf teil.

TOD WEGEN PERSONALMANGELS?

\section{Am Wochenende lieber keinen Infarkt}

Trotz aller interventionellen Aufrüstung der Kardiologie ist es nach wie vor gefährlicher, am Wochenende einen Infarkt zu bekommen als unter der Woche, wenn die Klinikteams vollzählig sind. „Am Wochenendeffekt hat sich nichts geändert, er persistiert, obwohl sich sonst die entsprechenden Parameter wie, Door-to-balloon'-Zeit und Mortalität in den letzten Jahren teilweise dramatisch signifikant verbessert haben“, sagte Abhishel Deshmukh vom Medical College of Wisconsin/USA. „Das kardiologische Team war an den Wochenenden nicht vollzählig verfügbar, in den meisten Kliniken oder Praxen war nur ein Kardiologe rufbereit."

In seiner Studie wertete Deshmuk 287470 Fälle aus. $22 \%$ davon wurden an einem Wochenende eingeliefert. Die Letalität am Wochenende lag deutlich höher als an einem Werktag $(7,58 \%$ versus $6,35 \% ; p<0,001)$. Wie zu erwarten erfolgten an den Wochenenden weniger Interventionen, und die verstrichene Zeit zwischen Einlieferung und Prozedur war länger.

\section{Vor allem jüngere Patienten betroffen}

Eine pathogenetische Wurzel für beide Erkrankungen wird in der vermehrten Entzündungsaktivität gesehen. Da vor allem jüngere Patienten betroffen sind, schlug Ahlehoff vor, das Management von Psoriasispatienten zu überdenken, vielleicht die Präventionsmöglichkeiten, etwa mit Statinen, großzügiger auszuschöpfen, als es für die Normalbevölkerung angeraten wird.

\section{INFARKTPRÄVENTION}

\section{Der „Koronare Stent-Pass“ ist da}

Bei der Behandlung von Patienten mit Koronarstents kommt es sehr auf die konsequente Einnahme von ASS und Clopidogrel an. Eine Unterbrechung der Therapie kann einen Infarkt auslösen. Die Deutsche Herzstiftung hat jetzt in Kooperation mit der DGK den „Koronaren StentPass“ vorgestellt, der Ärzte und Patienten bei der Kontrolle der Medikamenteneinnahme unterstützen soll. In den Ausweis kann der Arzt Stentart, Datum der Implantation sowie Art und Dosierung der Medikamente eintragen. Der „Koronare Stent-Pass" kann kostenlos per E-Mail unter info@herzstiftung.de bestellt werden. 